\title{
Improving self-efficacy in spinal cord injury patients through "design thinking" rehabilitation workshops
}

\author{
Daniel Wolstenholme, Tom Downes, Jackie Leaver, Rebecca Partridge, Joseph Langley
}

Sheffield Teaching Hospitals NHS Foundation Trust

\begin{abstract}
Advances in surgical and medical management have significantly reduced the length of time that patients with spinal cord injury (SCl) have to stay in hospital, but has left patients with potentially less time to psychologically adjust.
\end{abstract}

Following a pilot in 2012, this project was designed to test the effect of "design thinking" workshops on the self-efficacy of people undergoing rehabilitation following spinal injuries.

Design thinking is about understanding the approaches and methods that designers use and then applying these to think creatively about problems and suggest ways to solve them. In this instance, design thinking is not about designing new products (although the approaches can be used to do this) but about developing a long term creative and explorative mind-set through skills such as lateral thinking, prototyping and verbal and visual communication.

The principles of "design thinking" have underpinned design education and practice for many years, it is also recognised in business and innovation for example, but a literature review indicated that there was no evidence of it being used in rehabilitation or spinal injury settings.

Twenty participants took part in the study; 13 (65\%) were male and the average age was 37 years (range 16 to 72 ). Statistically significant improvements were seen for EQ-5D score $(t=-3.13, p=0.007)$ and Patient Activation Measure score $(t=-3.85, p=0.001)$. Other outcome measures improved but not statistically. There were no statistical effects on length of stay or readmission rates, but qualitative interviews indicated improved patient experience.

\section{Problem}

Advances in surgical and medical management have significantly reduced the length of time that patients with spinal cord injury (SCl) have to stay in hospital, but has left patients with potentially less time to psychologically adjust.

In 2012 The Princess Royal Spinal Injuries Unit, Sheffield was involved in a pilot "design and rehabilitation" project undertaken by Sheffield Hallam University's Lab4Living team supported by the Silvia Adams Trust and The Royal Society for the encouragement of Arts, Manufactures and Commerce(RSA). Both staff and participants had given favourable feedback on the experience.

This project builds on this work by aiming to explore if the sessions could allow patients with $\mathrm{SCl}$ to have a greater say in their own rehabilitation, and see what positive effect this could have on helping them to deal with the life-changing effects of SCl.

This project was set in a tertiary centre for spinal injuries in Sheffield, UK. The Princess Royal Spinal Injury Unit, Sheffield, is a supra-regional centre and is the second largest in the UK, catering to a population of around 9 to 10 million. It is a standalone, selfcontained unit, with its own staff and specialist facilities. The unit takes an average of 140 patients a year over four wards, acute, rehab, neuro-rehabilitation and readmission. The acute and rehab wards were involved in the project.

\section{Background}

There is increasing interest in the theory and practice of design in healthcare. Reports by UK organisations including NESTA [1], Demos [2], and The Design Council [3] discuss the need for and benefits of both using design methods and involving a wider group of patients, communities, and health professionals in the development of health services.

There are many drivers for health service reform: a rise of long term conditions, an aging population, a health service that has evolved to deliver acute care rather than primary care, reduced funding, and increasing expectations from an increasingly informed population. These are some of the key challenges to society today, and ones that require a new way of thinking. These need to be a radical step change in the ways we deliver care, with innovative approaches.

These innovative approaches have been explored through projects like user-centred healthcare design (UCHD).[4] UCHD was a fiveyear project funded by the United Kingdom National Institute for Health Research (NIHR) as part of the Collaboration for Leadership in Applied Health Research and Care (CLAHRC) for South Yorkshire that looked specifically at the use of design in health and social care. Work of the UK Design Council (and others) around 
"designing bugs out" [5] and reducing violence in A\&E have also worked specifically in this area.

Design thinking is the term given to designerly practice when undertaken by "non-designers." There are descriptions of design thinking being used in the healthcare setting since 2002 at the Mayo Clinic, North America, where physicians and designers have tested hypotheses about ways in which providers and patients interact.[7] Brown describes being involved in teaching design thinking to nurses, doctors, and administrators in the hope of inspiring practitioners to contribute new ideas to a project that lead to innovative changes within clinical practice.[8] However, in our literature review we found no reported intervention in the UK or internationally that applies design thinking in the context of teaching patients the skills involved in design thinking, besides the work that was conducted by the RSA in the pilot in 2010 .

In November 2010, The RSA Design \& Rehabilitation project was unique in piloting a workshop for eight people with spinal cord injuries, aiming to test the effectiveness of design training in developing resourcefulness and self-reliance. Campbell [9] suggested that if people (and not just designers) have tools available to be more resourceful, then changes may be seen in their self-management ability. The workshop anticipated that design thinking would foster independence by addressing the loss of confidence and motivation that often results from a sudden physical impairment.[10] Coleman [11] developed the idea of inclusive design, which forms a part of the theory for the original workshop. Inclusive design encourages designers to design for social inclusion, in which the output is not associated with negative perceptions of age or disability.

Anecdotally, some rehabilitation specialists recognise that there are many inpatient $\mathrm{SCl}$ patients who struggle with boredom and monotony of inpatient life, which can reinforce depression.[10] Having a design workshop that complements existing rehabilitation will stimulate patients who are mentally independent. Currently, published NICE guidelines do not exist for the rehabilitation of $\mathrm{SCl}$ patients and the National Spinal Cord Injury Strategy Board, lead by Dr Fiona Barr, are working towards developing SCI care pathways. Therefore it makes it a pertinent time to be evaluating the design thinking workshop and its impact on SCl patients.

\section{Baseline measurement}

To measure the impact of the workshops on patient self efficacy, the clinical psychologist from the unit was asked to identify several validated questionnaires to gather information pre and post intervention from our participants. These were the Perceived Manageability Scale (PMnac) and the Appraisals of Disability: Primary and Secondary Scale (ADAPSS), both of which are on the UK national dashboard as outcome measures for psychological interventions, and are validated for use in SCl. Following initial consultation the Patient Activation Measure (PAM) [6] was added as both predictor of compliance and outcome measure of the workshops.

Health economist advice was to use the EQ-5D quality of life questionnaire as this gives the greatest validity to compare across other interventions.

The EQ-5D-3L is a five item preference-based quality of life measure that can be used in economic evaluations to estimate quality adjusted life years (QALYs). The instrument asks five questions on mobility, self-care, usual activities, pain, anxiety, and depression, from which respondents are asked to choose three response options; no problems, some problems, or severe problems. Scores are converted into a score on a 0 to 1 scale, where 1 denotes full health and 0 death, and scores less than 0 denote states worse than death. Scores presented here are based on the York MVH tariff.[7] Participants also rated their health on a visual analogue scale ranging from 0 ; worst imaginable state to 100 best imaginable health state.

Paired t-tests were used to compare health related quality of life (HRQL) pre and post intervention. ANOVA was used to look for differences over time for ADAPSS and PMnac. Stata version 12 was used for all analysis.

Time series data were used to explore any potential impact on length of stay or emergency readmission rates, and the Trust's informatics team supported this. The challenges with getting the hospital activity data included: dates not properly recorded in medical notes, confusion over medically fit, and estimated dates of discharge, and skewed data by movement around wards. Due to these constraints the patient information systems in the Trust was used to look at the spell length of stay (LoS) by consecutive patient, for patients discharged from either of the wards where people were recruited.

The data collection time period was the 1st of January 2013 to 31 st March 2014 and patients whose date of discharge fell in that time period were identified. The SHINE intervention took place between June and December in this time period.

\section{Design}

Building on the workshops delivered as part of the RSA's pilot, a range of creative activities chosen to illustrate various parts of the "design process" were created. These were to take the form of group activities where two or more participants would spend an afternoon or morning with the design team to build up skills and understanding of design thinking.

The team had previous experience of delivering such workshops to a range of stakeholders, from professionals to young people, and knew that there was added value in working in groups. There was no-one with experience of delivering these workshops to inpatients.

There was initial success in finding like-minded people who undertook the sessions together. However, it became clear that this was unusual. Participants in subsequent group sessions had different approaches to learning, different levels of engagement, and different timetables for their traditional rehabilitation which made it difficult to find mutually acceptable time slots. 
The team met to discuss this and proposed a different approach based on their experience, moving to 1:1, 45 minute sessions integrated into their rehabilitation timetable. Again, the ability to adapt the nature and delivery of the intervention was an important consideration.

In the project the sessions were delivered by design researchers from Sheffield Hallam University Art and Design Research Centre who worked closely with the occupational therapists and other clinical staff to ensure that the workshops were appropriate from a clinical perspective.

The final version of the workshops comprised five topic areas, delivered over four to five, 45 minute sessions. The sessions worked through a series of activities based on the pilot project but developed by working with patients and staff over the first few months of the project. The sessions developed understanding in the patients about the design thinking approach and culminated in a practical "challenge" linked to the individuals own circumstances.

The workshops have been transformed into a tool kit with resources and instructions that can be used by any member of staff to deliver the sessions, as well as by volunteers such as those who work with the unit from the spinal injuries charities.

\section{Strategy}

The previous section described how we developed the initial idea into one that could be tested. This was an iterative process of learning through doing, and one guided by the participants of the study. The iterative design process used by designers is cyclical, like 'the model for improvements' PDSA improvement cycles, however the learning emerges from the relationship between designer and participant that develops through doing. The practice based nature of creative design also means that the studying and doing create embodied knowledge in the next version of the toolkit that is created, rather than setting off into a new PDSA cycle.

The final round of changes were made after the evaluation period and based on reflections from the team and participants. This final cycle of development resulted in the creation of the toolkit as a means of transferring the knowledge from the project to others.

\section{Results}

Twenty participants took part in the study $13(65 \%)$ were male and the average age was 37 years (range 16 to 72 ). For EQ-5D score and VAS and PAMS a higher score indicates better outcomes and this was the case for all three measures, with statistically significant improvements for EQ-5D score $(t=-3.13, p=0.007)$ and PAM $(t=$ $-3.85, \mathrm{p}=0.001$ ) (table 1).

The appraisals of disability scores (ADAPSS) and PMnac were filled in after each workshop. These are presented in table 2 below. There is no significant change in scores over time, however ADPASS scores after workshop 4 are significantly higher than those for workshops 1,2 , and 3 respectively (WS1 $t=-3.29, p=$
$0.004 ;$ WS2 $t=-4.12, p=0.001 ;$ WS $3 t=-3.37, p=0.004)$.

The two run charts above show NO3 (the rehab ward) and NO1 (the acute ward). Both show a relatively chaotic system in terms of spell LoS, however there appears to be a reduction in LoS (although not reaching the strict definition of a special cause variation) toward the end of November to December 2013. When speaking to staff on the unit they reported that there was a large waiting list for admission that built up over the summer period while the wards were closed for infection control requirements. This resulted in patients arriving on the unit already well advanced in their rehabilitation and therefore not requiring as much intervention and corresponding shorter LoS. For the purposes of the study the intervention did not appear to have an effect on the global LoS for patients through the unit, although this is a chaotic system and only a proportion of patients received the intervention.

In terms of readmission rate, the patient information system only identified 51 patients being readmitted within 28 days to the unit since 2006. In hindsight this was a redundant measure in this study. When analysed, the qualitative interviews showed two key themes around learning and experience. These are illustrated below with quotes from participants.

Patient learning: Design thinking is a long term shift in thought processes. At the end of the workshops we had positive interview feedback about a change in peoples thought patterns and approaches suggesting success. Participants stated that they had used the skills learned, some as a general change in mind-set, and others in more specific ways such as skills in directing care. One patient in particular managed to overcome a problem with part of a journey home overseas during the session.

"I used it when thinking around setting a routine at home, the activities give a framework."

Patient experience: The project had a positive effect on the quality of the service and the experience of patients; interviews show that participants felt it was a positive addition to their time on the unit viewing it as an additional rehabilitation therapy that provided mental stimulation currently not explicitly served on the unit. Participants stated that they would have liked more of the sessions.

"It was more about my mental recovery whereas everything else is about my physical recovery; it was the only really mental exercise I got to do in the hospital."

"Very enjoyable, it gave us an opportunity to think outside the box, which we hadn't done for a long, long, time..."

See supplementary file: ds3580.docx - "tables and figures"

\section{Lessons and limitations}

One of the most important lessons reinforced (it's not a new challenge) in this project is that of context. The intervention in its preliminary form was not sensitive to the context of the unit that we were trying to implement it in. It was only when the team were on 
BMJ Quality Improvement Reports

the ground and working that the context was fully apparent. This shows the benefit of a practice based approach to improvement and of the strength of prototyping. Without the practical experience the team would have not known the changes required to maximise the potential success of the implementation.

There were benefits from having the creative design perspective, not only through their unique skill set, but also from fresh eyes on to the context. The designers could be naive to the context and ask the questions that the members of the team, who are embedded in practice, might not see or ask. This led to a very patient centric approach which is reflected in the qualitative interviews.

A limitation of this project is that it was a feasibility study, and that while positive impacts on the quantitative measures were demonstrated there is no suggestion of causality. Further experimental type study design would be required to show that the intervention had an effect above and beyond normal care.

\section{Conclusion}

While the LoS and readmission rate showed no change, we might have hypothesised that a single intervention would not demonstrate a significant change in a complex system where many other factors influence going home.

The qualitative data demonstrated not only a benefit for people's experience of being in hospital, but also alluded to the potential for this to be a life skill to take forward after discharge.

The qualitative data was supported by the various psychological metrics used. The EQ5D, despite the challenges of this context, demonstrated a significant positive change over time, as did the PAM. The PAM in particular is a key measure in that the participants were more activated, more likely to think creatively about their futures, and more likely to set goals after the intervention. This has big implications in terms of the timing of rehabilitation interventions to get the best results, or the nature of interventions to move people to the point where they are likely to succeed in their rehabilitation. There is considerable clinical interest in this measure that is a direct result of this project.

The conclusions for clinical practice in spinal rehabilitation and other rehabilitation settings is that this approach is closely aligned to other self management approaches. The 2011 Health Foundation publication "Helping people help themselves" [14] presents the evidence for a continuum of self management practice, with the research suggesting that the proactive strategies work best. "Design Thinking" workshops would appear to align to the approaches that focus on both self efficacy and behaviour change, and that along with motivational interviewing this might be a new and novel approach to investigate further.

These interventions are complicated to investigate as they are by definition complex interventions, however this paper demonstrates that a mixed method approach to evaluating this intervention can yield positive results. It provides a strong baseline for further empirical research and hints at a degree of transferability of this intervention to other clinical areas.

\section{References}

1. Horne $\mathrm{M}$, Khan $\mathrm{H}$, and Corrigan P. People Powered Health 2013, available at: http://www.nesta.org.uk/library/documents/PPHforpplbyppl2. pdf

2. Parker S, Heapy J. The Journey to the Interface: How public service design can connect users to reform, London, UK: Demos. 2006. Available at: http://demos.co.uk/publications/thejourneytotheinterface

3. Cottam H, and Leadbeater C. RED Paper 01 Health: Cocreating Services, 2004.

4. User-centred Healthcare Design. www.uchd.org.uk (accessed 28th Aug 2014).

5. Design bugs Out https://www.gov.uk/government/uploads/system/uploads/att achment_data/file/215293/dh_131199.pdf (accessed 28th Aug 2014).

6. Reducing violence and aggression in A\&E http://www.designcouncil.org.uk/projects/reducing-violenceand-aggression-ae (accessed 28th Aug 2014).

7. Yale School of Management: Design and Social Enterprise Case Studies. Mayo Clinic: Design Thinking in Health Care. Available at http://nexus.som.yale.edu/design-mayo/ (accessed 13 July 2012).

8. Brown T. Design Thinking. Harvard Business Review June 2008; 84-95.

9. Campbell E. You know more than you think you do: design as resourcefulness and self-reliance. RSA Design \& Society, 2009.

10. Campbell E. Design \& Rehabilitation. A three-day workshop in design for people with spinal cord injuries. RSA Projects 2011.

11. Coleman R. THE CASE FOR INCLUSIVE DESIGN - AN OVERVIEW, Proceedings of the 12 Triennial Congress, The International Ergonomics Association and The Human Factors Association of Canada. 1994.

12. Patient Activation measure, Insignia Health. Available at http://www.insigniahealth.com/solutions/patient-activationmeasure (accessed 4 July 2014).

13. Dolan P. Modelling valuations for EuroQol health states. Medical Care 1997a; 35(11): 1095-108.

14. Health Foundation: Helping people help themselves, Health Foundation 2011, London.

\section{Declaration of interests}

None declared.

\section{Acknowledgements}

The project team would like to thank Sophie Payne, Mark Cobb, Sally Davies, Tracey Young, the staff and patients of the Princess Royal Spinal Injuries Unit Sheffield Teaching Hospitals NHS Foundation Trust 


\section{BMJ Quality Improvement Reports}

This article presents independent research funded by the Health Foundation and supported by the National Institute for Health Research Collaboration for Leadership in Applied Health Research and Care Yorkshire and Humber (NIHR CLAHRC YH). The views and opinions expressed are those of the authors, and not necessarily those of The Health Foundation, the NIHR, or the Department of Health. 